\title{
EDITORIAL
}

\section{Decoding the cryptic genomes of fungi: the promise of novel antibiotics}
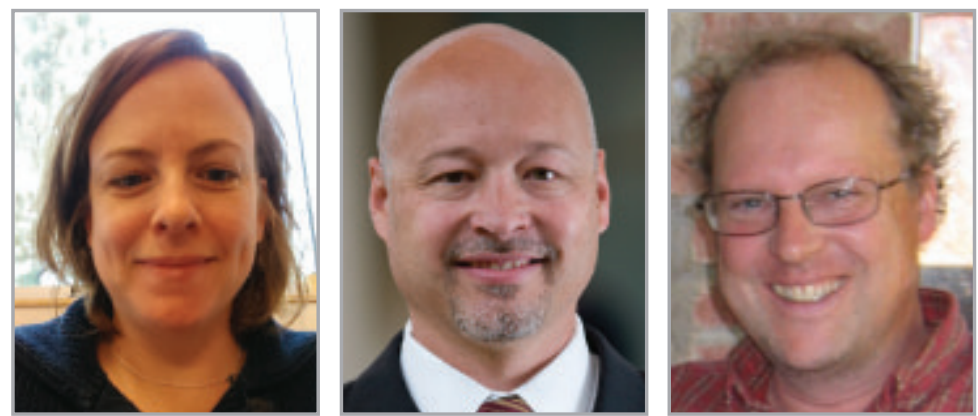

Kristina MSmith ${ }^{1,2}$, Jeffrey T Gautschi ${ }^{1,3}$ \& Michael Freitag ${ }^{*, 2}$

To humans, fungi are both friend and foe. While we have learned to harness their biochemistry to produce valuable foods, nutraceuticals and pharmaceuticals, fungi cause many devastating plant, animal and human diseases that result in billion-dollar costs annually worldwide. Filamentous fungi have been mined for natural products since the discovery of the first widely used modern antibiotic, penicillin, in 1929, as they are biochemically more interesting than their yeast cousins. Compounds essential for medicine include cyclosporin, gibberellins, cephalosporin, lovastatin, apicidin and numerous alkaloids. These diverse compounds are encoded by secondary metabolite (SM) gene clusters, which are regulated by environmental cues that often remain unknown. Unlocking the full potential of the many fungal genomes that have recently been sequenced is one challenge for natural product discovery in the coming years.

An untapped wealth of products is encoded in the many newly identified fungal SM gene clusters $[1,2]$, constituting 'cryptic genomes' - segments of DNA likely never expressed in the laboratory before - with potential for novel chemistry that may yield new antibiotics or other useful natural products. Development of new compounds to control multidrug-resistant pathogenic bacteria, as well as eukaryotic pathogens and parasites, is recognized as an urgent necessity. Recent comprehensive reports $[3,4]$ underscore the importance of re-emerging or newly emerging threats to human health globally. It is imperative that we discover and bring to market new compounds that fill the widening gap between resistant organisms [5-7] and current therapies.

For many years the hurdle in discovering metabolites produced from novel SM clusters in filamentous fungi has been to find conditions that induce gene expression. The 'one strain many compounds' (OSMAC) concept, investigating multiple, sometimes extreme, growth conditions to induce production of a variety of metabolites from single fungal strains is successful, although labor intensive [8]. Attempts at

\section{KEYWORDS}

- antibase $\bullet$ Aspergillus $\bullet$ fungi

- Fusarium • histone methylation

- natural products $\bullet$ Neurospora

- secondary metabolites

"To humans, fungi are both friend and foe. While we have learned to harness their biochemistry to produce valuable foods, nutraceuticals and pharmaceuticals, fungi cause many devastating plant, animal and human diseases...” 
“The emphasis should be on finding novel chemistry rather than taking incremental steps towards new compounds." overexpressing specific transcription factors or expression of entire clusters in bacteria or yeast also requires significant input of time and labor for the uncertain return of potentially discovering one new metabolite [2]. This proved truly slow work even in the genus Streptomyces, long considered the workhorse of novel antibacterial production. In fact, only two new classes of antibiotics have reached the market in the past 40 years, daptomycin from Streptomyces [9] and the synthetic oxazolidinones [10]. With now more than 600 genomes of diverse filamentous fungi in hand, discovery of novel compounds from this group is in full swing. Work in the genus Aspergillus has progressed most rapidly and resulted in the identification of several global regulators that are required for the expression of SMs [1,2]. Inhibition of epigenetic regulators mostly failed to increase global expression of SM, although individual clusters are often expressed, as illustrated by use of the histone deacetylase inhibitor suberoylanilide hydroxamic acid for production of a novel cyclodepsipeptide, EGM-556 [11].

The discovery of a global repressor of SM gene cluster expression, the H3K27 methyltransferase KMT6 [12], promises to overcome some of the problems mentioned above, at least in many fungi. H3K27 trimethylation is found in most Fusarium SM gene clusters and functions to silence expression during growth in the laboratory, usually in rich carbon and nitrogen sources and without competing microorganisms, thus mimicking conditions when SMs are not needed in nature. Removing the repressor by deletion of the $k m t 6$ gene resulted in increased expression of approximately 2000 genes, most of which encode proteins of unknown function. KMT6-mediated regulation appears to be conserved in many fungi. How can we make the best use of this genome-wide overexpression system to find useful natural products? What are the major challenges in harnessing novel metabolic pathways in fungi to find novel antibiotics?

\section{Annotation of SM gene clusters}

Each Fusarium species contains between 10 and 15 unique gene clusters encoding polyketide synthases or nonribosomal peptide synthases that make unknown products [13,14]. Overall, annotation of these SM gene clusters has progressed smoothly; however, only Aspergillus SM gene clusters are truly comprehensively annotated [1]. Much remains to be discovered, as bioactive compounds may be produced from 'mini clusters', genes encoding modifying enzymes (e.g., methyltransferases or CYP450s) that alter intermediate metabolites and that are often near genes encoding transporters that export natural products into the environment. Single genes encoding small peptides have largely unknown function and hundreds of these unstudied genes exist in each species. Considering that $20-25 \%$ of natural products possess some biological activity [15], taking into account the hundreds of new transcripts generated by the Fusarium graminearum kmt6 mutant in a single-culture medium tested [12], and the many strains of fungi that produce SMs, high-throughput screens for antibiotic activity promise good returns for investments.

\section{Identification of novel compounds}

Medium- to high-throughput pipelines for the discovery of bioactive compounds have been used for natural product discovery in prokaryotes. The emphasis should be on finding novel chemistry rather than taking incremental steps towards new compounds. Identification and disabling of global repressors such as KMT6 provides a novel approach, and it stands to reason that a high percentage of SM produced from genomes heretofore unexpressed will be novel, decreasing the probability of finding already known chemistry and speeding up discovery. Our HPLC separation of extracts from culture filtrate of the F. graminearum kmt 6 mutant showed dozens of peaks that were not present in extracts from the wild-type. Some of the conventional difficulties in natural product isolation will now need to be confronted, including the need for robust dereplication procedures, increased material or analytical tools for small amounts of material, and the avoidance of resource-intensive isolation of nonbioactive compounds.

Luckily, instrumentation, chemical databases, prefractionation methodologies and bioassay-guided isolation techniques have been greatly enhanced since the zenith of early antibiotic programs. Dereplication is the ability to identify known chemistry prior to embarking on any isolation efforts. Predicting compounds from fungal genomes alone is challenging; thus, dereplication is accomplished on crude extracts by use of liquid chromatography (LC) coupled to instruments that yield structural information, such as mass spectrometry (MS) 
and nuclear magnetic resonance (NMR), and a zoo of hyphenated approaches based on instrumentation (e.g., LC-MS, LC-MS-MS, LC-MS-ELSD-UV and LC-NMR) is available. Chemical masses and formulas from MS or NMR (helpful with more material and in lesscomplex mixtures) provide input for dereplication by comparison to large compound databases. These structure- and property-based searchable databases (e.g., antiBASE [16] and Dictionary of Natural Products [17]) have been vastly improved over the last 20 years and contain more than 100,000 entries; thus, investigators armed with reliable molecular weights and a chemical formula can routinely identify known chemistry. When there is ambiguity owing to multiple formulas matching one chemical mass, dereplication matches are vetted by overlaying species information and additional chemical data, such as UV spectrometry, NMR or MS-MS. Resources for computational dereplication of mixtures of natural products are continually improving, based on studies on marine organisms, bacteria and fungi [18-20].

\section{Bioactivity screens}

Most large pharmaceutical companies have abandoned programs that focus on antibiotic development; thus, screening for activity against pathogens occurs increasingly in university laboratories or startup companies. No standardized pipelines exist, and while individual publications may report on activity against specific organisms, screening is rarely comprehensive. It is thus desirable to reach a consensus on which pathogens should be part of screening panels and how screens should be conducted. For example, crude fractions from column chromatography or HPLC can be assessed for antibacterial activity on strains expressing fluorescent proteins or other quantifiable bacterial growth indicators in 96-well plates in automated setups. To reduce overall costs, decrease duplicated effort and increase safety, it appears logical to centralize screening in a few federally or foundation-funded cooperative facilities.

\section{Expression of cryptic clusters at levels required for structural studies $\&$ for production}

Once novel compounds have been identified, they need to be produced at levels sufficient for detailed structural studies and activity assays with purified compounds. The field has always been dogged by small amounts of available materials; however, without enough material at the end of multistep isolation procedures it is impossible to determine the final structure as well as the biological activity and safety of pure compounds. Advances in small-sample MS and NMR instrumentation now allow detection at the femtomolar and nanomolar levels, allowing investigators to identify novel chemistry.

Unlike some sources of natural products, many fungi are easily grown in large fermentors under a wide variety of conditions. Coupling this to genetic manipulations that increase production of many metabolites at one time, as in the case of the $k m t 6$ mutant, suggests a future where we may grow fungi in continuous culture to extract common but also low-abundance compounds from culture filtrates in successive isolation steps, first for purification for structural analyses and later in large-scale production setups. This will likely require expression of genes or gene clusters in heterologous hosts, now usually yeast. Different taxa of fungi have quite different biochemistry. Consequently, we should select host species with similar metabolic pathways that allow robust expression of specific gene clusters, which requires development of several filamentous fungi as industrial overexpression systems instead of relying exclusively on yeast. For the genus Aspergillus the candidate is Aspergillus niger, which can produce astonishingly large amounts of secreted proteins and other natural compounds. This species may also become the workhorse for expression of gene clusters from the related genus Penicillium. For Fusarium, one candidate species would be Neurospora crassa, perhaps the best-understood filamentous fungus, and one species in which few SM gene clusters are encoded and expressed, even in a kmt6 mutant [21].

\section{Last, but not least: funding}

While there are technical challenges to overcome, the major hurdle is a lack of funding. Since most large pharmaceutical companies have divested themselves of antibiotics divisions, innovative funding approaches are needed to promote research in the field. Governmental support, engagement by philanthropic foundations and start-up companies are keeping the field alive. Incentives for companies to enter the market will be necessary, while simple maximization of returns for newly developed products must be limited to keep medicines affordable.

\author{
"As academia and \\ nonprofit organizations \\ carry a larger burden, they \\ need to collaborate with \\ interests in emerging \\ economies to produce \\ medicines cheaply and \\ market them at more \\ reasonable margins."
}


As academia and nonprofit organizations carry a larger burden, they need to collaborate with interests in emerging economies to produce medicines cheaply and market them at more reasonable margins. We can also hope that humans will realize that access to effective treatments is a global human rights issue and therefore should not be reduced to maximizing income for stockholders. However we go about it, we need to restart the stalled engine of antibiotic discovery.

\section{Financial \& competing interests disclosure}

The authors have no relevant affiliations or financial involvement with any organization or entity with a financial interest in or financial conflict with the subject matter or materials discussed in the manuscript. This includes employment, consultancies, honoraria, stock ownership or options, expert testimony, grants or patents received or pending, or royalties.

No writing assistance was utilized in the production of this manuscript.

\section{References}

1 Inglis Do, Binkley J, Skrzypek MS et al. Comprehensive annotation of secondary metabolite biosynthetic genes and gene clusters of Aspergillus nidulans, A. fumigatus, A. niger and A. oryzae. BMC Microbiol. 13, 91 (2013).

2 Wiemann P, Keller NP. Strategies for mining fungal natural products. J. Ind. Microbiol. Biotechnol. 41(2), 301-313 (2013).

3 Antibiotic Resistance Threats in the United States, 2013. CDC, GA, USA (2013).

4 Laxminarayan R, Duse A, Wattal C et al. Antibiotic resistance-the need for global solutions. Lancet Infect. Dis. 13(12), 1057-1098 (2013).

5 Honda H, Dubberke ER. The changing epidemiology of Clostridium difficile infection. Curr. Opin. Gastroenterol. 30(1), 54-62 (2014).

6 Kester JC, Fortune SM. Persisters and beyond: mechanisms of phenotypic drug resistance and drug tolerance in bacteria. Crit. Rev. Biochem. Mol. Biol. doi:10.3109/1 0409238.2013.869543 (2013) (Epub ahead of print).

7 Salgame P, Yap GS, Gause WC. Effect of helminth-induced immunity on infections with microbial pathogens. Nat. Immunol. 14(11), 1118-1126 (2013).
8 Bode HB, Bethe B, Hofs R, Zeeck A. Big effects from small changes: possible ways to explore nature's chemical diversity. Chembiochem 3(7), 619-627 (2002).

9 Fenton C, Keating GM, Curran MP. Daptomycin. Drugs 64(4), 445-455; discussion 457-448 (2004).

10 Diekema DJ, Jones RN. Oxazolidinone antibiotics. Lancet 358(9297), 1975-1982 (2001).

11 Vervoort HC, Draskovic M, Crews P. Histone deacetylase inhibitors as a tool to up-regulate new fungal biosynthetic products: isolation of EGM-556, a cyclodepsipeptide, from Microascus sp. Org. Lett. 13(3), 410-413 (2011).

12 Connolly LR, Smith KM, Freitag M. The Fusarium graminearum histone H3 K27 methyltransferase KMT6 regulates development and expression of secondary metabolite gene clusters. PLoS Genet. 9(10), e1003916 (2013).

13 Brown DW, Butchko RA, Baker SE, Proctor $\mathrm{RH}$. Phylogenomic and functional domain analysis of polyketide synthases in Fusarium. Fungal Biol. 116(2), 318-331 (2012).

14 Wiemann P, Sieber CM, Von Bargen KW et al. Deciphering the cryptic genome: genome-wide analyses of the rice pathogen Fusarium fujikuroi reveal complex regulation of secondary metabolism and novel metabolites. PLoS Pathog. 9(6), e1003475 (2013).
15 Demain AL, Sanchez S. Microbial drug discovery: 80 years of progress. J. Antibiot. (Tokyo) 62(1), 5-16 (2009).

16 Laatsch H. AntiBase 2013: The Natural Compound Identifier. Wiley Press, NY, USA (2013).

17 Dictionary of Natural Products on DVD (Version 19.1). Buckingham J (Ed.). Chapman and Hall/CRC, FL, USA (2013).

18 Lang G, Mayhudin NA, Mitova MI et al. Evolving trends in the dereplication of natural product extracts: new methodology for rapid, small-scale investigation of natural product extracts. J. Nat. Prod. 71(9), 1595-1599 (2008).

19 Johnson TA, Sohn J, Inman WD et al. Natural product libraries to accelerate the high-throughput discovery of therapeutic leads. J. Nat. Prod. 74(12), 2545-2555 (2011).

20 El-Elimat T, Figueroa M, Ehrmann BM, Cech NB, Pearce CJ, Oberlies NH. High-resolution MS, MS/MS, and UV database of fungal secondary metabolites as a dereplication protocol for bioactive natural products. J. Nat. Prod. 76(9), 1709-1716 (2013).

21 Jamieson K, Rountree MR, Lewis ZA, Stajich JE, Selker EU. Regional control of histone H3 lysine 27 methylation in Neurospora. Proc. Natl Acad. Sci. USA 110(15), 6027-6032 (2013). 